\title{
On the time-scales of the downward propagation and of the tropospheric planetary wave response to the stratospheric circulation
}

\author{
G. Nikulin ${ }^{1, *}$ and F. Lott $^{1}$ \\ ${ }^{1}$ Laboratoire de Météorologie Dynamique, UMR 8539, Paris, France \\ *now at: Swedish Meteorological and Hydrological Institute, Norrköping, Sweden
}

Received: 28 July 2009 - Revised: 30 November 2009 - Accepted: 18 January 2010 - Published: 1 February 2010

\begin{abstract}
Three datasets (the NCEP-NCAR reanalysis, the ERA-40 reanalysis and the LMDz-GCM), are used to analyze the relationships between large-scale dynamics of the stratosphere and the tropospheric planetary waves during the Northern Hemisphere (NH) winter. First, a crossspectral analysis clarifies the time scales at which downward propagation of stratospheric anomalies occurs in the lowfrequency band (that is at periods longer than 50 days). At these periods the strength of the polar vortex, measured by the 20-hPa Northern Annular Mode (NAM) index and the wave activity flux, measured by the vertical component of the Eliassen-Palm flux $(\mathrm{EPz})$ from both the troposphere and the stratosphere, are significantly related with each other and in lead-lag quadrature. While, in the low-frequency band of the downward propagation, the EPz anomalies of the opposite sign around NAM extremes drive the onset and decay of NAM events, we found that the EPz anomalies in the troposphere, are significantly larger after stratospheric vortex anomalies than at any time before. This marked difference in the troposphere is related to planetary waves with zonal wavenumbers $1-3$, showing that there is a tropospheric planetary wave response to the earlier state of the stratosphere at low frequencies. We also find that this effect is due to anomalies in the EPz issued from the northern midlatitudes and polar regions.
\end{abstract}

Keywords. Meteorology and atmospheric dynamics (General circulation; Middle atmosphere dynamics; Waves and tides)

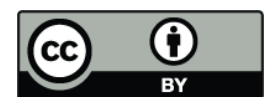

Correspondence to: G. Nikulin (grigory.nikulin@smhi.se)

\section{Introduction}

In the Northern Hemisphere (NH) middle and polar latitudes, the stratospheric variability is very pronounced during the winter months. This follows from the fact that during this period, the stratospheric winds are eastward, which permits planetary Rossby waves (zonal wavenumber 1-3) to propagate into the stratosphere (Charney and Drazin, 1961). The interaction between these waves and the large-scale flow can lead to very large changes in the mean stratospheric circulation. As planetary waves entering into the stratosphere have their origin in the troposphere, it seems reasonable to consider that the stratospheric variability is essentially a response to the tropospheric variability. This view is confirmed by the diagnostic study in Newman et al. (2001) and which shows that the anomalous state of the stratospheric polar vortex depends on the Wave Activity Flux (WAF), approximated by zonal mean eddy heat flux, entering into the stratosphere from the troposphere during a preceding period. In Newman et al. (2001), the periods considered typically exceed a month, with stratospheric anomalies in midwinter, affecting the stratospheric temperature in early spring.

Over the last 15 years, a large number of studies have also suggested that an anomalously strong or weak stratospheric polar vortex influences the tropospheric circulation at a later stage. The low-frequency stratospheric anomalies, measured by the Arctic Oscillation (AO) index or by the zonal-mean zonal wind $(\bar{u})$, often propagate downward at the tropopause level, and sometimes deeper into the troposphere reaching the surface (Kodera et al., 1990; Baldwin and Dunkerton, 1999; Christiansen, 2001). Again, the time scales concerned are around a month and even more, with Kodera et al. (1990) using a one month boxcar average to establish their results, Baldwin and Dunkerton (1990) a 90day low-pass filter, and Christiansen (2001) a 30-360 day band-pass filter. Using comparable techniques and filters, the

Published by Copernicus Publications on behalf of the European Geosciences Union. 
low-frequency downward propagation in the stratosphere is also found in GCMs (Christiansen, 2001; Lott et al., 2005). The understanding of the stratospheric vacillations has been advanced by model studies such as Holton and Mass (1976), Schoeberl (1983), Christiansen (1999), Plumb and Semeniuk (2003) and Scott and Polvani (2004). They prove that the stratospheric vacillations originate from local wave-mean flow interactions, somehow reminiscent of the dynamics of the Quasi-Biennal Oscillation (QBO) in the tropical stratosphere. Interestingly, these authors have also shown that changes in the tropospheric circulation are not needed to produce the stratospheric low frequency vacillations: if the tropospheric forcing is not changed, the EP-fluxes entering in the stratosphere still vary according to the changes in the stratospheric circulation.

The significance of this problem for the surface climate was established by Thompson et al. (2002), and Polvani and Waugh (2004) who found anomalies in the surface weather a few weeks and more after the appearance of strong anomalies in the stratospheric circulation. The origin of this tropospheric signal has been much debated. It can be related to the $\mathrm{AO}$ descent down to the tropopause, as suggested by Baldwin and Dunkerton (1999), but one needs to understand how this signal in the low stratosphere affect the weather in the low troposphere. Among the mechanisms proposed, some are rather direct, like the remote tropospheric signature of stratospheric potential vorticity anomalies (Black, 2002; Ambaum and Hoskins, 2002), or like so called the downward control (Haynes et al., 1991; Thompson et al., 2006). Besides those "direct" effects on the troposphere, various numerical and observational studies have shown that the baroclinic instability life cycle in the troposphere is sensitive to the state of the stratosphere, and in particular to the vertical shear of the zonal wind $\left(\bar{u}_{z}\right)$ in the lower stratosphere (Tanaka and Tokinaga, 2002; Song and Robinson, 2004; Wittman et al., 2004, 2007; Limpasuvan et al., 2004, 2005).

Another possible mechanism, is that the upward propagating planetary waves that themselves drive the stratosphere, can be reflected downward, hence modifying the planetary wave activity in the troposphere (e.g., Perlwitz and Harnik, 2003). In support with this, Coughlin and Tung (2005) have shown that the quasi-stationary planetary waves in the troposphere are also sensitive to the stratospheric conditions, so they can also respond to downward propagating stratospheric anomalies in zonal-mean zonal wind $\bar{u}$. Note here that the various mechanisms listed here and before are not exclusive with each other, with the "downward control" effect for instance, and the effect due to the planetary waves reinforcing each other in Song and Robinson (2004).

In a comparable line of work, Karpetchko and Nikulin (2004) have found substantial anticorrelations between the WAF averaged in time over November-December (ND, or early winter in the following) and the WAF averaged in time over January-February (JF or midwinter). In their study the WAF essentially represents the conventional Eliasen-Palm
(EP) flux vector $\left(F^{(\phi)}, F^{(z)}\right)$, but their results mainly concern its vertical component $\left(F^{(z)}\right)$. They also found an increase in the midwinter tropospheric $F^{(z)}$ when the JanuaryFebruary polar night jet is stronger than usual and opposite. However, they did not detail to which extent the changes in the stratospheric circulation produce the changes in the WAF at a later stage. On top of this, they again implicitly used ad-hoc low pass filters, somehow hiding the ranges of frequencies at which an effect on the planetary waves due to the stratospheric circulation can be seen at a latter stage.

Because this is today a central problem in our understanding of the climate, and because each authors have used different time filters, it seems worthwhile to clarify the timescales at which the AO can be seen as propagating downward and to verify that these time-scales correspond to the time-scales at which the WAF and the AO interact with each other. This is the first objective of this paper and we emphasize here that these time scales are not related to the time it takes for anomalies to propagate from the stratosphere to the troposphere. As the reanalysis datasets are not entirely consistent from a dynamical viewpoints, we will use for this purpose two of them, the NCEP/NCAR reanalysis and the ERA40 reanalysis, and we will also use a 20 -year integration done with the LMDz-GCM. The second objective of this paper is to verify that at these frequencies, there is a lowfrequency relationship between the stratospheric circulation and the tropospheric planetary waves at a latter stage. We will also verify that the mechanisms suggested in the past to explain this stratospheric influence on the planetary waves, are consistently found at the time-scales and lags identified in the study.

The plan of the paper is as follows. Section 2 presents the datasets and the methods. The characteristic frequencies for the downward propagation and for the coupling between the NAM index and the WAF at different levels are analyzed by a cross-spectral analysis in Sect. 3. Section 4 identifies the tropospheric planetary wave response to the stratospheric circulation examining the difference between preceding and subsequent wave forcing about extremes in the strength of the stratospheric polar vortex. The analysis in Sect. 4 focuses on the low-frequency intraseasonal band revealed by the spectral analysis in Sect. 3, and using a low-pass filter appropriate for this frequency band. The physical mechanisms responsible for the planetary waves response to the stratospheric circulation are discussed in Sect. 5. Our main results are summarized in Sect. 6 .

\section{Data and method}

The first database we use is the daily NCEP-NCAR reanalysis for the 1978-2005 period when satellite data is included in the reanalysis (Kalnay et al., 1996). To verify that the results obtained do not depend on the database chosen, we have also used the daily ERA-40 reanalysis for the 1978-2002 
period (Uppala et al., 2005). Since the results are identical, the results from the NCEP-NCAR reanalysis are only presented here. Finally, we also perform the same analysis on the stratospheric version of the LMDz General Circulation Model presented in Lott et al. (2005). For completeness note that the LMDz GCM is a gridpoint model in the horizontal direction with a uniform resolution of $2.5^{\circ}$ in latitude and $3.75^{\circ}$ in longitude. The vertical resolution is in term of a hybrid sigma-pressure vertical coordinate with 50 levels and an upper boundary at near $65 \mathrm{~km}$. The model results presented are from a 20 year integration forced at the lower boundary by sea surface temperature and sea ice cover that only vary along a climatological annual cycle.

For all datasets, the daily anomalies are calculated by subtracting the daily climatological annual cycle. The variability of the zonal mean state is characterized by the NAM pattern defined at each level as the leading empirical orthogonal function (EOF) of the monthly-mean zonal-mean geopotential height anomalies north of $20^{\circ} \mathrm{N}$. The daily NAM index is then constructed by projecting daily zonal-mean geopotential height anomalies onto the leading EOF pattern. A positive value of the NAM index in the stratosphere corresponds to a stronger than usual polar night jet. This approach is similar to methodology suggested by Baldwin and Thompson (2009). Only difference is that the EOFs are based on monthly means here and on daily means in Baldwin and Thompson (2009) but we found that the results are not sensitive to a choice of monthly or daily means for calculation of the EOFs. The quasigeostrophic Eliassen-Palm flux in spherical coordinates $F=\left(F^{(\phi)}, F^{(z)}\right)$ is used as a measure of the wave activity flux (Edmon et al., 1980; Andrews et al., 1987). As an integral measure of wave fluxes for the $\mathrm{NH}$ we use the EP flux components averaged over the $45^{\circ}-75^{\circ} \mathrm{N}$,

$$
\left\langle F^{(\phi, z)}\right\rangle=\int_{45}^{75} F^{(\phi, z)} \cos \phi d \phi,
$$

where $\phi$ is the latitude.

To focus attention on the variability of the atmosphere in specific frequency bands, we will apply a finite impulse response filter based on the sinc function. The filter kernel $h_{\mathrm{f}}$ is given by

$h_{\mathrm{f}}(i)=\frac{\sin (2 \pi f i)}{2 \pi f i}, \quad-N \leq i \leq N$,

where $f$ is the cutoff frequency (or the half power point in the spectral domain), and where $2 N+1$ is the filter length (Smith, 2002). The filter length is chosen equal to the cutoff period in days. This is an optimal choice, yielding to a rather sharp transition in the frequency domain near $f$ but resulting in small overshoots and ringings in the step response. To minimize the Gibbs effect the filter kernel is multiplied by a Kaiser window with a sidelobe attenuation of $50 \mathrm{~dB}$ (Thede, 2004).
To determine the frequency band at which the NAM index and the EP flux are related with each other, we compute the coherence and phase spectra between them. The coherence spectrum is dimensionless and analogous to the conventional squared correlation coefficient that depends upon frequency. To evaluate these spectral estimates we use a "chunk" spectral estimator in the terminology of von Storch and Zwiers (1999, p. 270) and first focus onto the NH winter period and divide the data into non-overlapping samples of equal length (365 days) beginning at 1 July and ending at 30 June next year. Each sample is detrended and a cosine bell taper is applied to the first and last 90 points. For each samples of the NAM and the EP fluxes we take the Fourier transform and evaluate the individual periodograms and crossperiodograms for each year. The spectral $\left(\hat{\Gamma}_{\mathrm{NAM}}\right.$ and $\left.\hat{\Gamma}_{\mathrm{EP}}\right)$ and cross-spectral $\left(\hat{\Gamma}_{\mathrm{NAM}, \mathrm{EP}}=\hat{\Lambda}_{\mathrm{NAM}, \mathrm{EP}}+i \hat{\Psi}_{\mathrm{NAm}, \mathrm{EP}}\right)$ estimates are obtained by averaging the individual periodograms and cross-periodograms over all years. From these spectral estimates we derive the coherence and the phase by

$$
\begin{aligned}
& \hat{k}_{\mathrm{NAM}, \mathrm{EP}}=\frac{\hat{\Gamma}_{\mathrm{NAM}, \mathrm{EP}}^{2}}{\hat{\Gamma}_{\mathrm{NAM}} \hat{\Gamma}_{\mathrm{EP}}} \\
& \hat{\Phi}_{\mathrm{NAM}, \mathrm{EP}}=\tan ^{-1}\left(\frac{\hat{\Psi}_{\mathrm{NAM}, \mathrm{EP}}}{\hat{\Lambda}_{\mathrm{NAM}, \mathrm{EP}}}\right),
\end{aligned}
$$

respectively (for details see von Storch and Zwiers, 1999, p. 222-287).

The statistical significance for the coherence spectra are determined by a Monte-Carlo method. The Monte-Carlo test uses 500 pair of an autoregressive process of order 1 (AR1) with variance and lag 1 autocorrelation estimated from the original data. In each pair, the AR1 surrogates are independent of each other, hence, the test for the coherency spectrum evaluates in which frequency band the original series are significantly related. For each surrogate pair the coherency spectrum is estimated exactly as it is for the original series. For each frequency, this yields to 500 estimates of the coherency between two unrelated AR1 processes, and from these 500 values we collect statistics for the $95 \%$ and 99\% confidence levels. Although this method does not take into account the fact that in the original data most of the variability occurs during the winter season we have verified that weighting the artificial time series by an annual cycle for the variance little affect our test (see also Christiansen, 2001). For the phase, we compute the confidence intervals using $t$ statistics (e.g. von Storch and Zwiers, 1999, p. 285). Dividing the original data into 27 samples gives approximately 54 degrees of freedom to build this statistics.

We use a similar method to compute and test the cross correlations and the lag regressions between the filtered time series. First, a filter is applied to the original data, and then the filtered data is divided into non-overlapping "winter" samples beginning at 1 October and ending at 30 April next year. Each sample is detrended, individual cross correlations or lag 

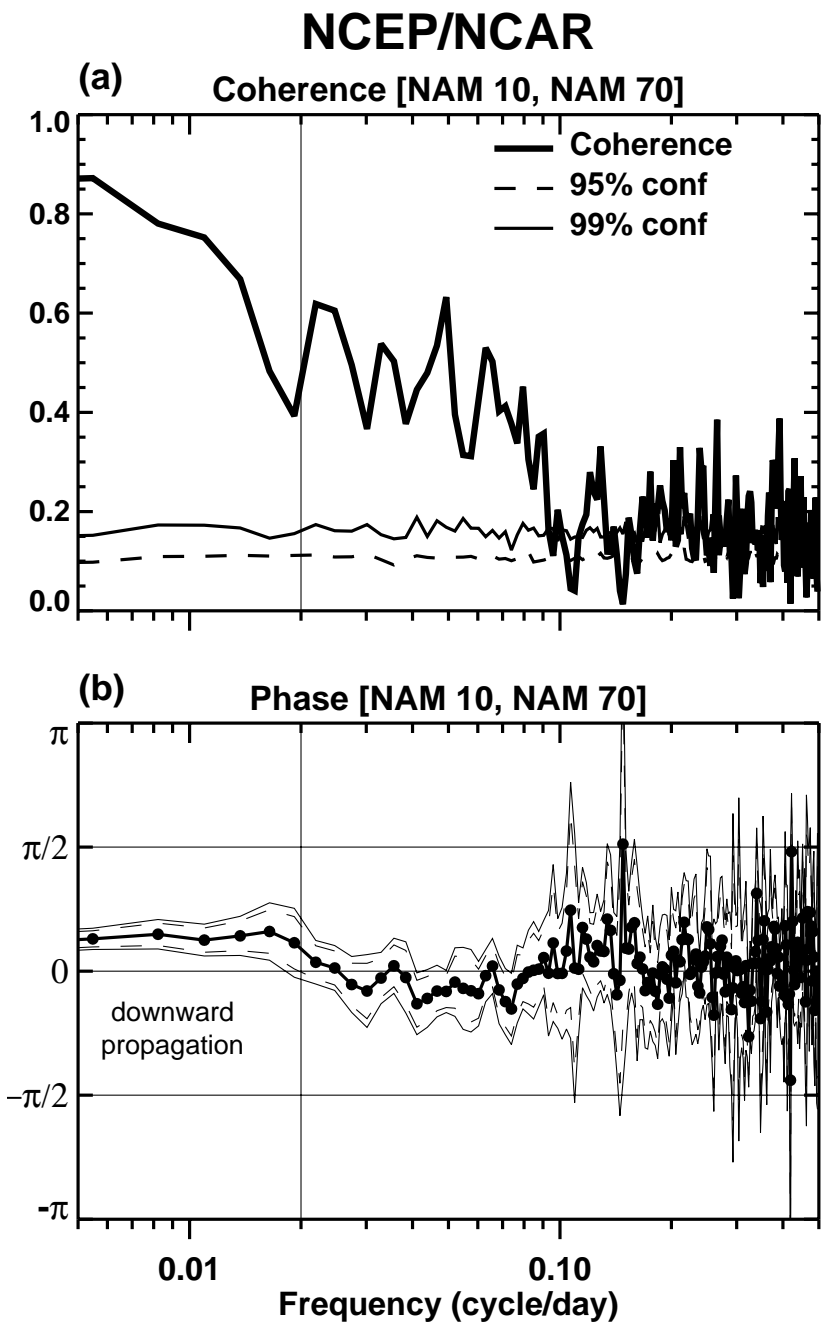

Fig. 1. Cross-spectral analysis between the NAM index at $10 \mathrm{hPa}$ and $70 \mathrm{hPa}$. Dashed and thin solid lines are the $95 \%$ and $99 \%$ confidence levels, respectively. The NCEP-NCAR reanalysis.

regressions are then calculated and averaged. The same sequence is applied to 500 pair of low-pass filtered AR1 surrogates independent of each other, and to estimate the $5 \%$ and $1 \%$ significance levels by a Monte-Carlo test.

\section{Downward propagation and NAM-EP flux coupling}

To determine the characteristic frequencies of the downward propagation in the stratosphere Fig. 1 presents the coherence and phase spectra between the NAM indices at 10 and $70 \mathrm{hPa}$. These two levels are chosen here for convenience, and because the signature of the downward propagation clearly appears between them. For the NCEP-NCAR reanalysis, the coherence between the NAM indices at 10 and $70 \mathrm{hPa}$ is significant for almost all periods (Fig. 1a) and substantially increases when the period increases. According to the phase spectrum in Fig. 1b the coherence spectrum
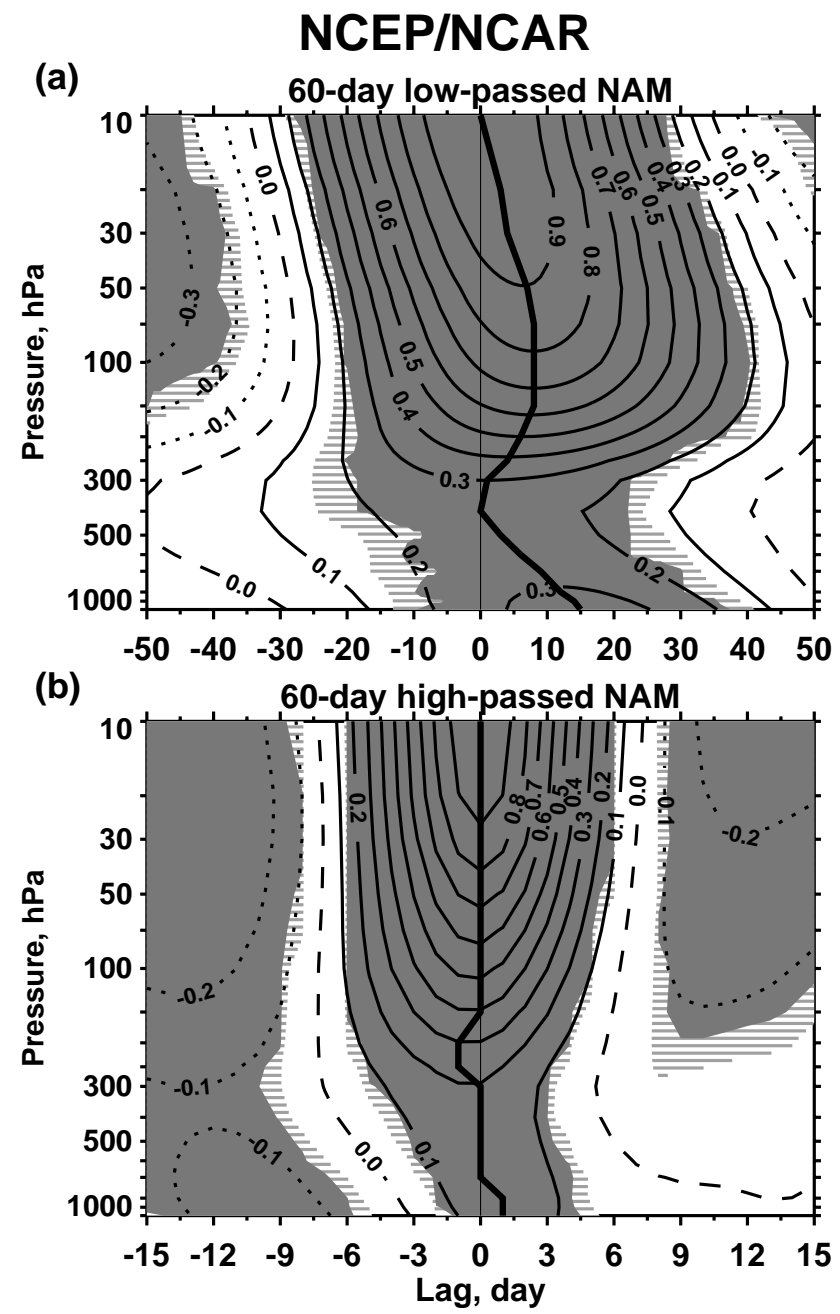

Fig. 2. Cross correlation of the NAM index at $10 \mathrm{hPa}$ with the NAM index at each level for (a) the 60-day low-passed NAM index and (b) the 60-day high passed NAM index. Positive contour values are solid, negative values are dotted and zero contours are dashed. The $5 \%$ (lines) and $1 \%$ (solid) significance levels are shaded. The thick black line is the position of the maximum cross correlation at each level. The NCEP-NCAR reanalysis.

can be separated into two frequency bands. The first one is at periods longer than 50-60 days where the phase is positive thus the signal at $10 \mathrm{hPa}$ leads the signal at $70 \mathrm{hPa}$ indicating downward propagation. A cross-correlation analysis of the 60-day low-passed NAM index (Fig. 2a) reveals that the downward propagation of the NAM signal from $10 \mathrm{hPa}$ into the lower stratosphere takes about 10 days and then a weak signal appears near the ground with a maximum at 15-day lag. The second band is approximately between 10 and 50 days where the phase has a tendency to be negative but the magnitude is hardly different from zero. A crosscorrelation analysis of the 60-day high-passed NAM index (Fig. 2b) indeed shows that the high-passed NAM index is in phase at all levels i.e. there is no the downward propagation 


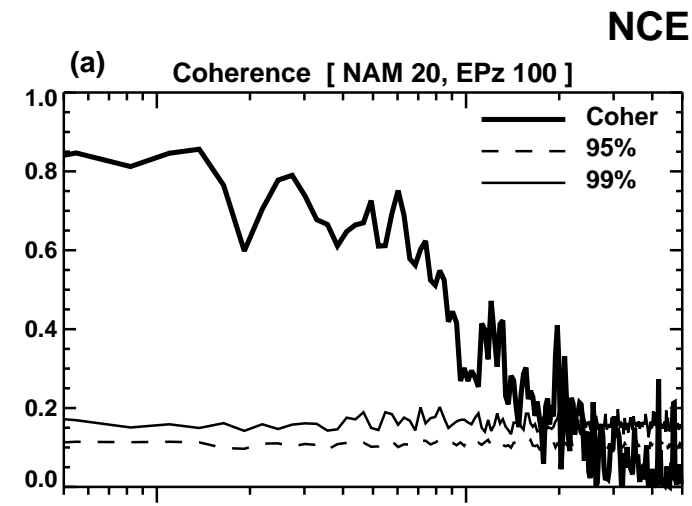

NCEP/NCAR
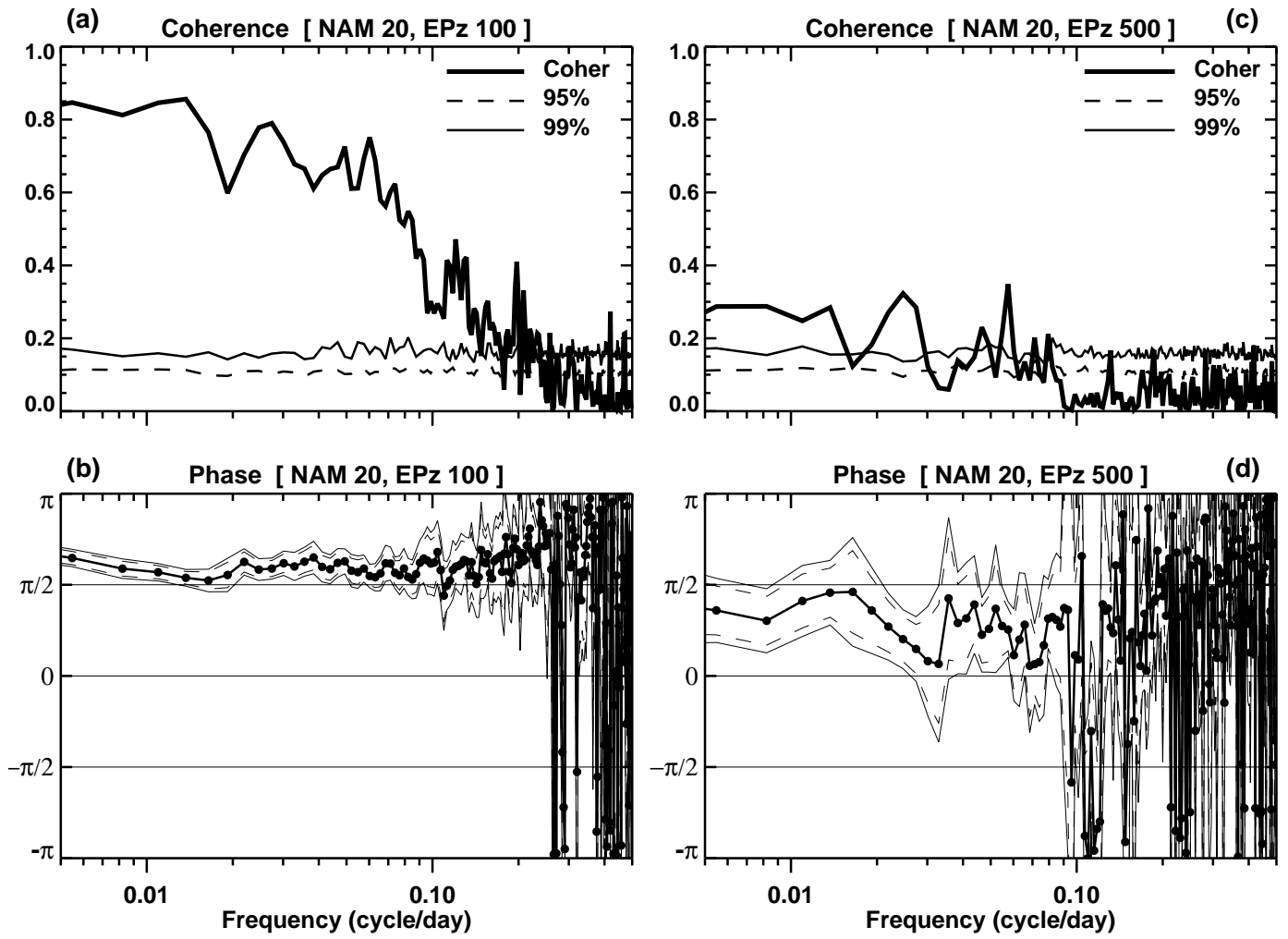

Fig. 3. Cross-spectral analysis between the NAM index at $20 \mathrm{hPa}$ and the vertical EP flux anomalies averaged over $45^{\circ}-75^{\circ} \mathrm{N}$ at $($ a, b) $100 \mathrm{hPa},(\mathbf{c}, \mathbf{d}) 500 \mathrm{hPa}$. Dashed and thin solid lines are the $95 \%$ and $99 \%$ confidence levels respectively. The NCEP-NCAR reanalysis.

of stratospheric anomalies at these frequencies. If we apply the same cross-spectral analysis for levels below $70 \mathrm{hPa}$ the coherence at low frequencies decreases, completely disappearing at $300 \mathrm{hPa}$, and then weakly significant coherence appears near the ground. Thus the cross-spectral and crosscorrelation analysis clearly shows that the downward propagation in the stratosphere occurs at periods longer than 50-60 days. We will next focus on the wave-mean flow interactions in this low-frequency band.

To investigate this in more detail we next proceed to a cross-spectral analysis between the the NAM at $20 \mathrm{hPa}$ and the EP flux in the lower stratosphere at $100 \mathrm{hPa}$ and in the middle troposphere at $500 \mathrm{hPa}$, averaged over $45^{\circ}-75^{\circ} \mathrm{N}$ (e.g. $\left\langle F^{(\phi, z)}\right\rangle$ ). Similarly to the coherence between the NAM indices in Fig. 1a, the coherence between the NAM index at $20 \mathrm{hPa}$ and the vertical EP flux $\left\langle F^{(z)}\right\rangle$ at $100 \mathrm{hPa}$ in Fig. 3a increases when the frequency decreases. The corresponding phase (Fig. 3b) is positive and close to $90^{\circ}$. Similar results are found when $\left\langle F^{(z)}\right\rangle$ is taken at other levels in the lower stratosphere above $100 \mathrm{hPa}$ (not shown). When the averaged vertical EP flux $\left\langle F^{(z)}\right\rangle$ is evaluated below $100 \mathrm{hPa}$, the coherence decreases, but remains significant in several frequency bands, including the low-frequency band of the downward propagation at periods longer than 50 days (see for instance Fig. 3c). The phase at low frequencies is positive and significantly different from zero in both the stratosphere (Fig. 3b) and the troposphere (Fig. 3d), with $\left\langle F^{(z)}\right\rangle$ being almost in lead-lag quadrature with the NAM index. This lead-lag quadrature indicates a significant relationship where $\left\langle F^{(z)}\right\rangle$ is the forcing and the NAM is the response: during the NAM life-cycle the wave forcing anomalies lead the NAM anomalies of the opposite sign and follow the NAM anomalies of the same sign (see for example Christiansen, 2001; Limpasuvan et al., 2004, 2005). The same characteristic time scales of the downward propagation and the high coherence between the NAM and $\left\langle F^{(z)}\right\rangle$ in the stratosphere support the previous observational and model studies (Christiansen, 2001; Plumb and Semeniuk, 2003) suggesting that the downward propagation in the stratosphere is driven by low-frequency wave forcing through local wave-mean flow interaction.

In opposite to $\left\langle F^{(z)}\right\rangle$ the coherence between the NAM index at $20 \mathrm{hPa}$ and the horizontal EP flux $\left\langle F^{(\phi)}\right\rangle$ at $100 \mathrm{hPa}$ is largest at synoptic time scale (Fig. 4a). There is also a significant coherence in the low-frequency band, at periods longer than 100 days. In the middle troposphere (Fig. 4c) the coherence substantially decreases at synoptic time scale but increases at low frequencies. At these low frequencies, 


\section{NCEP/NCAR}
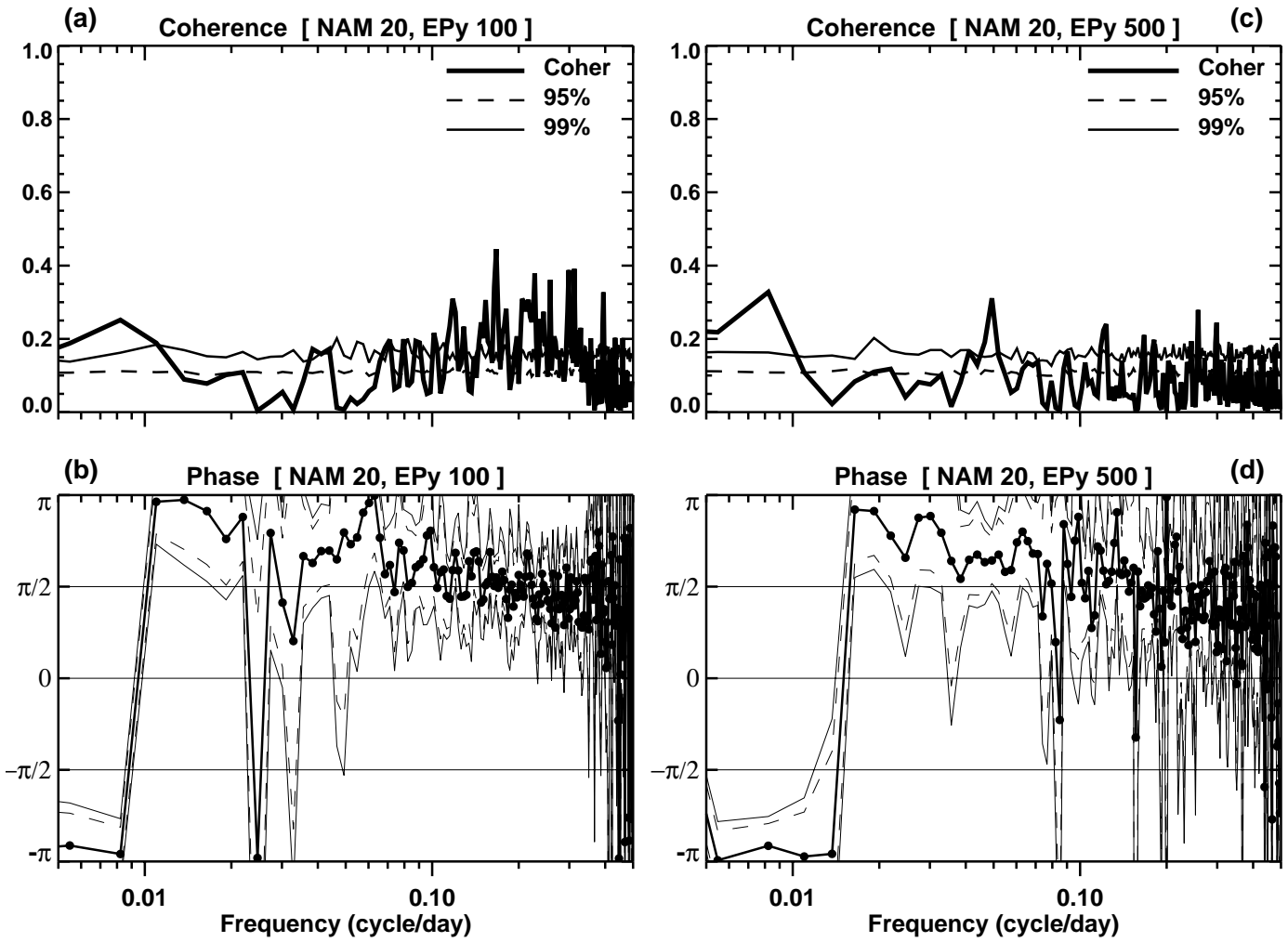

Fig. 4. As Fig. 3, but for the horizontal EP flux.

in both the stratosphere and the troposphere, the phase between two signals is close to $180^{\circ}$ or $-180^{\circ}$ (Fig. 4b, d), so the NAM and $\left\langle F^{(\phi)}\right\rangle$ are in antiphase. In this case the NAM modulates the horizontal EP flux and the $\left\langle F^{(\phi)}\right\rangle$ anomalies almost coincide to the NAM anomalies of the opposite sign as, for example, shown by Limpasuvan et al. $(2004,2005)$ using composite analysis.

The cross-spectral analysis applied to the 20-year simulation with the LMDz GCM (not shown) gives results which are almost identical to the one applied to the NCEP-NCAR reanalysis. The model reproduces well the phase and coherence between the 20-hPa NAM and the EP fluxes in both the stratosphere and the troposphere at periods longer than 5060 days, as well as the downward propagation at the same periods. Hence the low-frequency stratospheric behaviour and the downward propagation in the LMDz GCM are essentially a response to the low-frequency variability of the wave forcing, as in the reanalyses.

While the response in the tropospheric horizontal EP flux $\left\langle F^{(\phi)}\right\rangle$ to stratospheric conditions is more straightforward and well documented (Limpasuvan et al., 2004, 2005) the response in the tropospheric vertical EP flux is more difficult to define. The $\left\langle F^{(z)}\right\rangle$ anomalies following the NAM extremes are an integral part of the NAM lifecycle, namely they drive the decay of the NAM events but they cannot be directly in- terpreted as stratospheric influence of the NAM on the tropospheric $\left\langle F^{(z)}\right\rangle$. To isolate if such an influence exist, we will next focus on the low-frequency vertical EP flux anomalies around NAM extremes and more specifically on the difference between the preceding and subsequent $\left\langle F^{(z)}\right\rangle$ anomalies in the troposphere.

\section{NAM: preceding and subsequent wave forcing}

\subsection{December-January NAM}

To analyse the differences between the wave property before and after stratospheric zonal mean circulation anomalies, we first follow in part Karpetchko and Nikulin (2004) and evaluate the one point correlations between the December-January (DJ) NAM index at $20 \mathrm{hPa}$ and the ND $F^{(z)}$. We then repeat the same one-point correlation with the DJ NAM but using the JF $F^{(z)}$. For the preceding period and for the NCEPNCAR reanalysis in Fig. 5a, we find a negative correlation in most places in the troposphere and in the stratosphere. In contrast, for the subsequent period in Fig. 5c, the correlation is predominantly positive. This result is consistent with the fact that strong (weak) $F^{(z)}$ precedes a weak (strong) polar vortex and then a weak (strong) $F^{(z)}$ follows. We should note that the correlation pattern in Fig. $5 \mathrm{c}$ is almost identical 

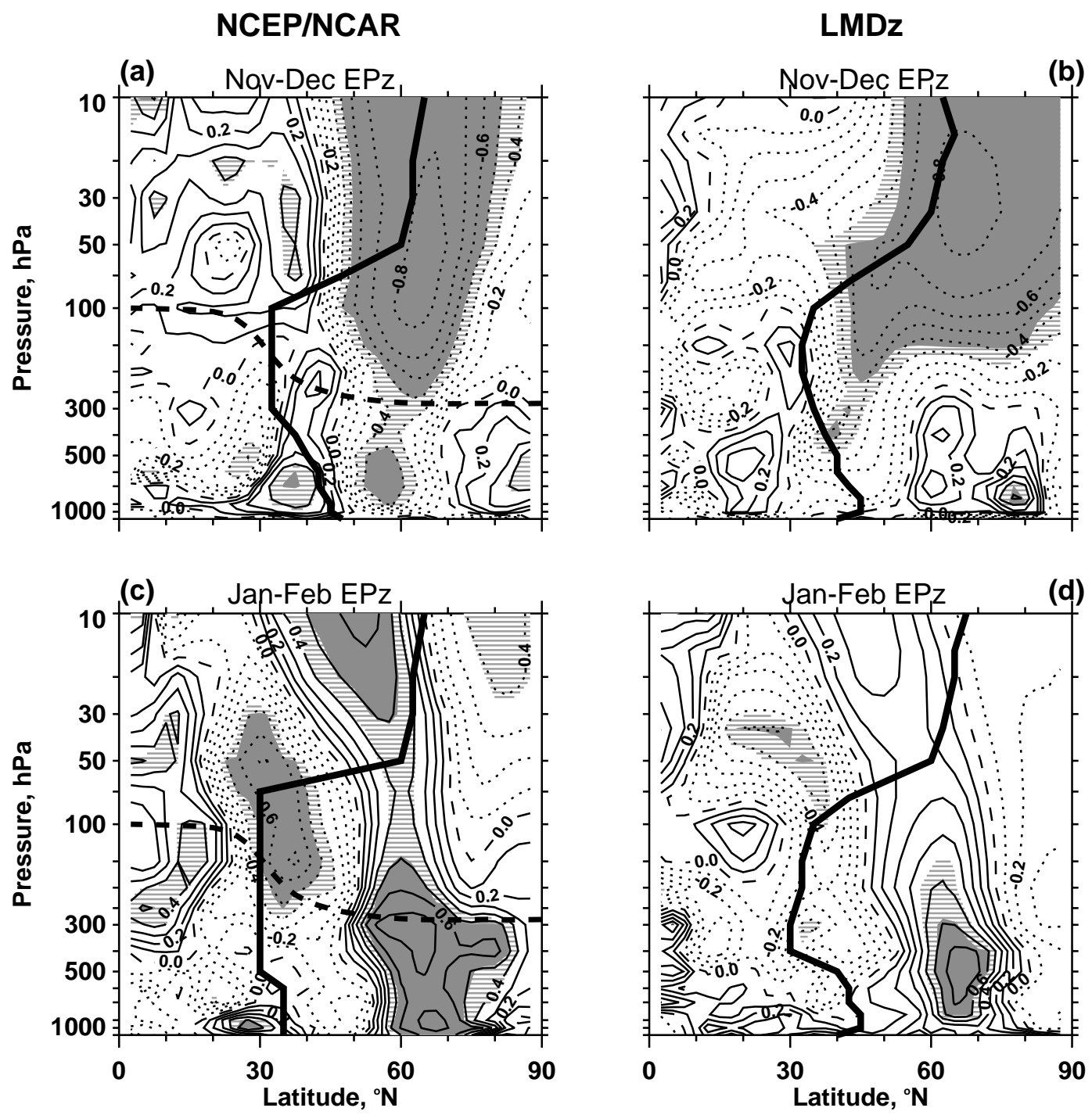

Fig. 5. Correlation between the December-January NAM index at $20 \mathrm{hPa}$ and the vertical EP flux $\left(F^{(z)}\right)$ anomalies. (a-b) $F^{(z)}$ is averaged over November-December; (c-d) $F^{(z)}$ is averaged over January-February; (a-c) NCEP-NCAR reanalysis; and (b-d) LMDz-GCM. The thick black line is the position of the maximum zonal mean zonal wind at each level and the dashed line is the tropopause. Contours and shading as in Fig. 2.

to the one obtained in Karpetchko and Nikulin (2004), where the correlation is keyed to the ND eddy heat flux at $20 \mathrm{hPa}$ averaged over $45^{\circ}-75^{\circ} \mathrm{N}$ rather than over the DJ NAM and where the JF eddy heat flux is shown (see their Fig. 1a). Our results here prove that the anticorrelation between the early winter wave forcing and the midwinter wave forcing found by these authors is associated with anomalies in the stratospheric NAM occurring between these periods.

If we now examine the Fig. 5a and c with more details and in the stratosphere, we can notice that they present noticeable difference in the lat-altitude distribution of the vertical EP flux. In the stratosphere, the ND $F^{(z)}$ in Fig. 5a is anticorrelated with the 20 -hPa NAM over a large domain $\left(50^{\circ}-80^{\circ} \mathrm{N}\right)$ corresponding to the stratospheric jet. On the contrary, and still in the stratosphere, the correlation pattern between the 20-hPa NAM and the JF $F^{(z)}$ has a dipole structure with positive and negative correlation located equatorward and poleward of the stratospheric jet respectively. These differences in the spatial distribution of the correlations are related to the existence of the polar and midlatitude waveguides (Dickinson, 1968; Nikulin and Karpechko, 2005). Depending on the high-latitude structure of zonal wind after a NAM event, upward propagating waves from the troposphere can be refracted either to the midlatitude stratosphere (a strong polar night jet) or to the polar stratosphere (a weak polar night jet).

If we move down to the troposphere, the differences between the preceding period and the subsequent period are even more pronounced. In particular, in the subsequent 

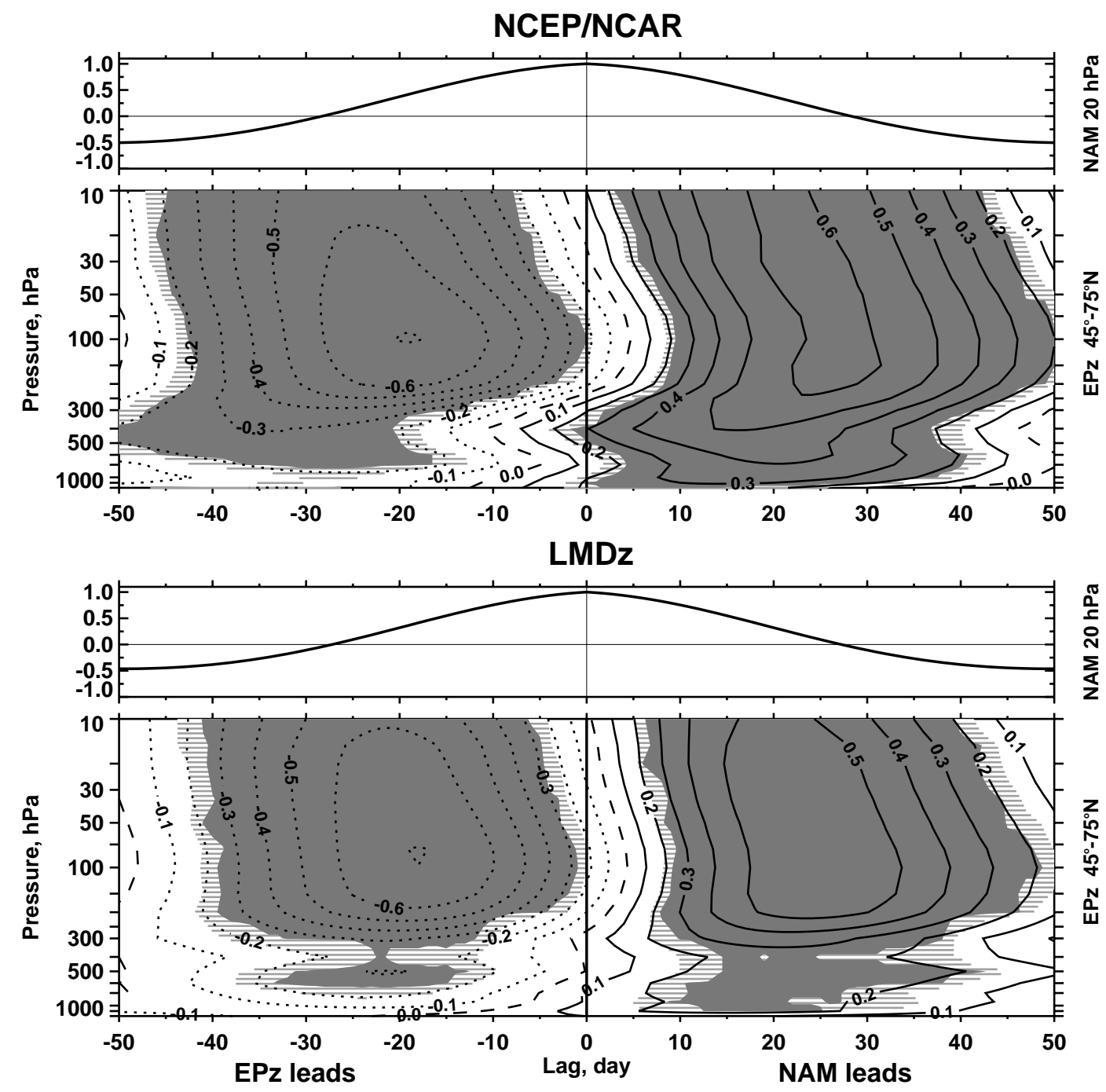

Fig. 6. Cross correlation of the 60-day low-passed NAM index at $20 \mathrm{hPa}$ with itself and with the 60-day low-passed vertical EP flux anomalies averaged over $45^{\circ}-75^{\circ} \mathrm{N}$. The (top two plots) NCEP-NCAR reanalysis and the (bottom two plots) LMDz GCM. Contours and shading as in Fig. 2.

period in Fig. $5 \mathrm{c}$ and between $60^{\circ}$ and $80^{\circ} \mathrm{N}$, there is a strong tropospheric correlation with the stratospheric NAM, which has almost no counterpart of opposite sign in Fig. 5a, that is in early winter. Since the middle and lower stratosphere is much more disturbed after a $20-\mathrm{hPa}$ NAM event, this difference in the correlation patterns suggests that the stratospheric circulation affects the tropospheric $F^{(z)}$ at a later stage.

The same one-point correlations for the LMDz GCM are shown in Fig. 5b, d. The LMDz GCM reproduces that in ND the negative correlation in the extratropical stratosphere (Fig. 5b) is stronger in amplitude than the opposing positive correlation found in JF (Fig. 5d). Note also, that in the LMDz GCM, there is a strong tropospheric correlation in midwinter (JF, Fig. 5d) which has no opposing counterpart in early winter (ND, Fig. 5b). Although less pronounced than in the reanalysis, this difference in the troposphere suggests that there is a dynamical influence of the stratosphere on the troposphere in the model as well.

Finally, to determine which waves are responsible for the $F^{(z)}$ correlation in Fig. 5, we have partitioned the total $F^{(z)}$ into $F^{(z)}$ due to planetary-scale waves (zonal wavenumber 13 ) which dominate in the stratosphere, and $F^{(z)}$ due to the smaller-scale waves $4-7$ which play a role in the troposphere. This partitioning shows that the waves 1-3 are the main contributor to the observed correlation patterns at least north of $45^{\circ} \mathrm{N}$ for both the reanalysis and the LMDz GCM. Only the negative correlation in the subtropical lower stratosphere for JF (Fig. 5c and d) is related to the waves 4-7. 

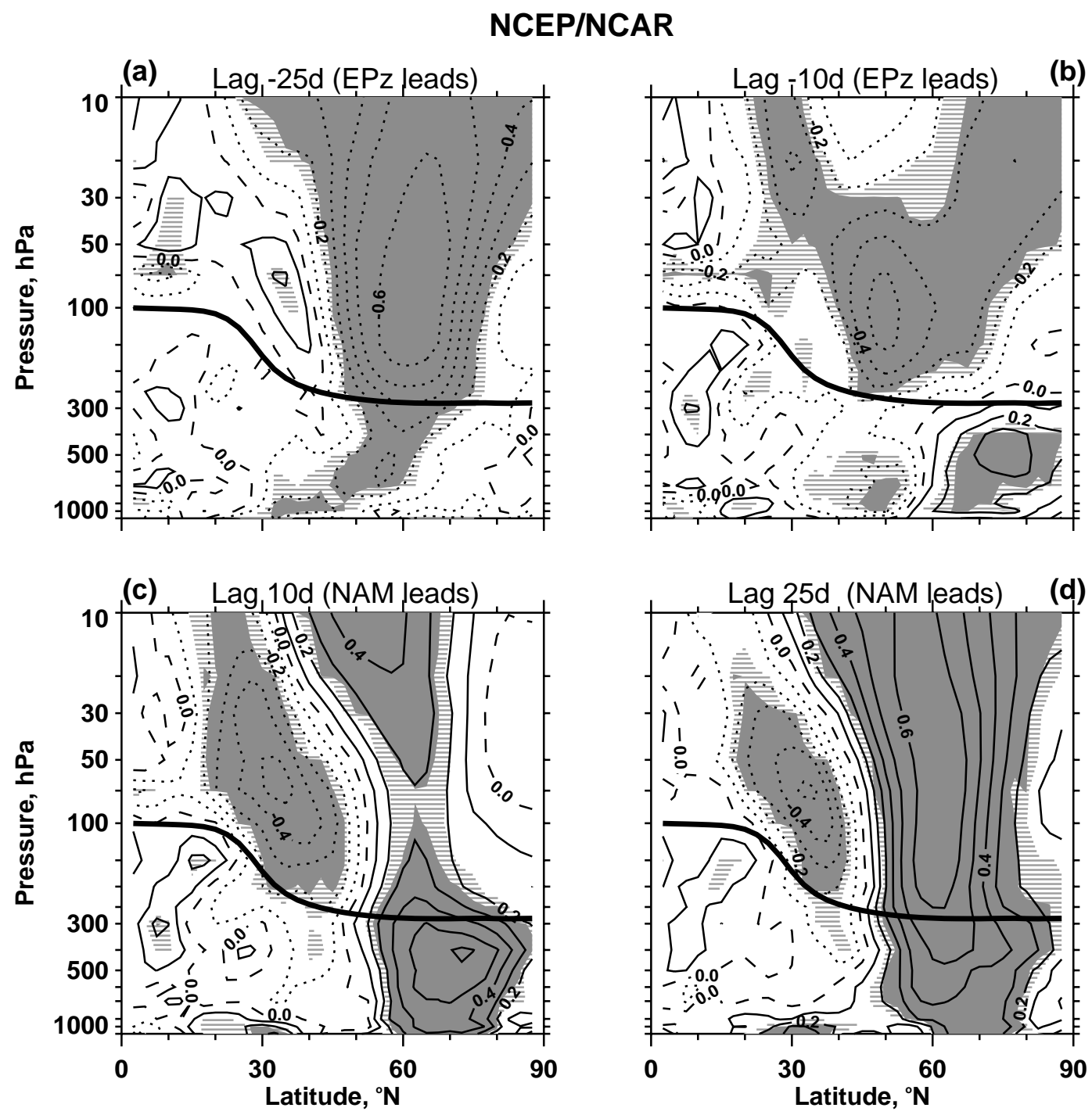

(b)

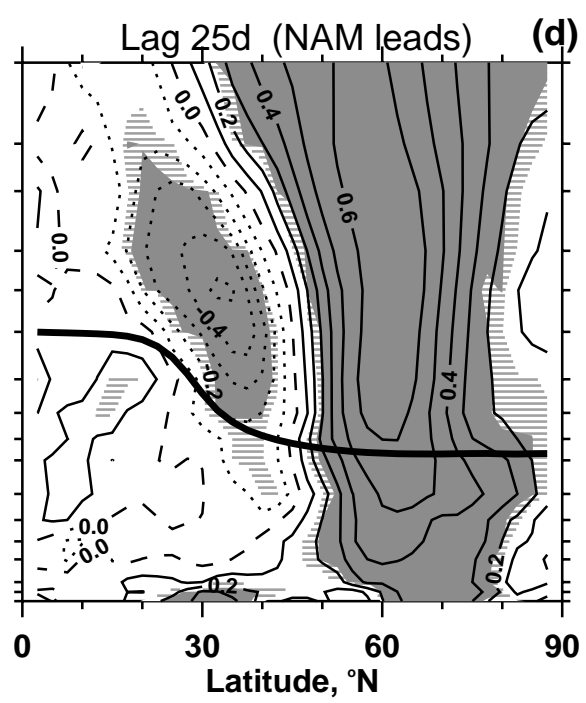

Fig. 7. Cross correlation of the 60-day low-passed NAM index at $20 \mathrm{hPa}$ with the 60 -day low-passed vertical EP flux anomalies. Crosscorrelations are taken at lags (a) -25 , (b) -10 , (c) 10 and, (d) 25 days. Contours and shading as in Fig. 2. The NCEP-NCAR reanalysis.

\subsection{Daily low-passed NAM}

The two-month boxcar average used in Fig. 5 is equivalent to applying a low-pass filter with half-power point at 100 days. It therefore cuts off a considerable part of the spectrum where the coherences seen in Fig. 3 are significant, that is for all the periods longer than 10 days. It also excludes a part of the downward propagating NAM signal which starts to propagate downward at periodicities longer than 50 days (see the thin vertical line in Fig. 1b). To capture both effects more completely, and also to select the whole winter period for the NAM than JF only, we next filter all our daily series with the 60-day low-pass filter given in Eq. (2).

Figure 6 shows the cross correlation of the daily NAM index at $20 \mathrm{hPa}$ with itself (thick lines in the top panels) and with the daily vertical EP flux averaged over $45^{\circ}-75^{\circ} \mathrm{N}$ $\left(\left\langle F^{(z)}\right\rangle\right)$. Again, the reanalysis (top panels in Fig. 6) and the LMDz GCM (bottom panels in Fig. 6) are in good agreement with each other. In the stratosphere the strongest negative correlations are observed when the EP flux $\left\langle F^{(z)}\right\rangle$ leads the NAM index by about 20 days, while the strongest positive correlations are found when $\left\langle F^{(z)}\right\rangle$ follows the NAM index by about 25 days. In the troposphere, the correlations in Fig. 6 are in general weaker than the stratospheric ones, but the values are still significant at both positive and negative lags. Note nevertheless, that the tropospheric correlations at positive lags are much larger in amplitude than those at negative lags, which is not the case in the stratosphere.

To determine the spatial distribution of the wave response, Fig. 7 presents the cross correlation of the 20-hPa NAM with $F^{(z)}$ in the latitude-pressure plane. For the NCEP-NCAR reanalysis the correlations are about maxima in the stratosphere 


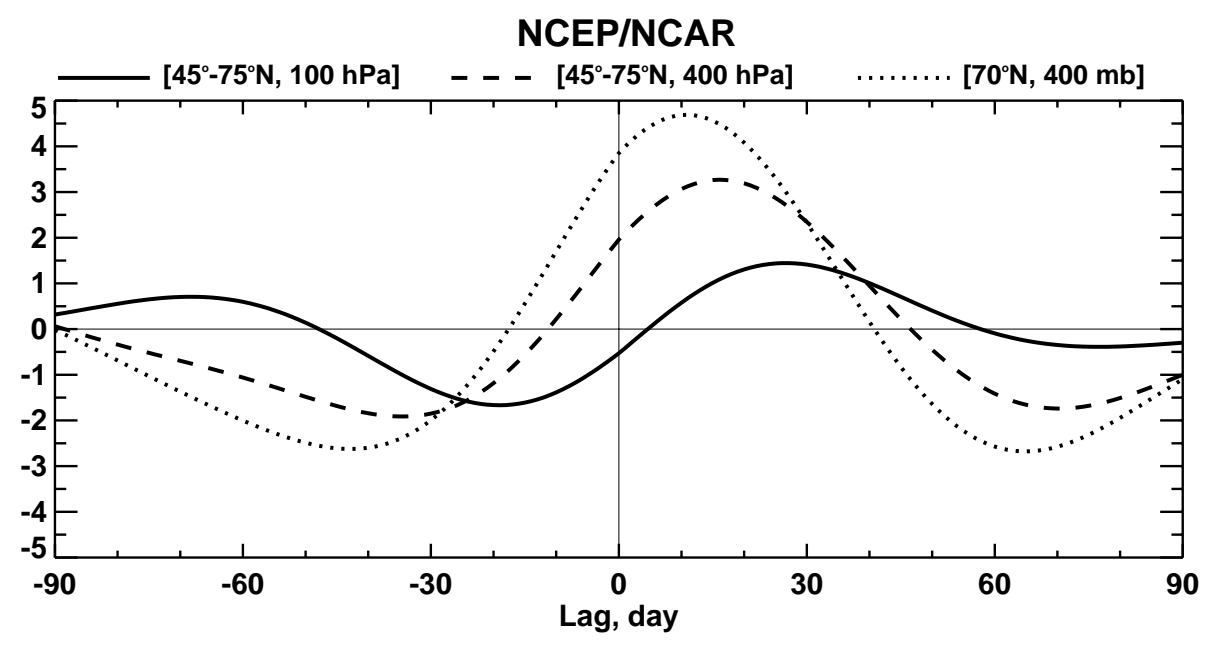

Fig. 8. Lagged regression of the 60-day low-passed normalized NAM index at $20 \mathrm{hPa}$ on the 60-day low-passed vertical EP flux anomalies taken at (solid) $100 \mathrm{hPa}$ over $45^{\circ}-75^{\circ} \mathrm{N}$, (dotted) $400 \mathrm{hPa}$ over $45^{\circ}-75^{\circ} \mathrm{N}$ and, (dashed) at $400 \mathrm{hPa}, 70^{\circ} \mathrm{N}$. Units are $10^{4} \mathrm{~kg} \mathrm{~m}{ }^{-1} \mathrm{~s}^{-2}$. The NCEP-NCAR reanalysis.

at lags -25 days (Fig. 7a) and 25 days (Fig. 7d), in agreement with the results in Fig. 6. In the stratosphere, the maximum absolute value of correlation at negative lag is around 0.6 , and almost equal to the value at positive lag. On the contrary, the correlation in the troposphere at positive lag is very pronounced in the polar latitudes while at negative lag the correlation in the troposphere is much smaller (Fig. 7ab). In the troposphere the signal appears near lag -10 days north of $70^{\circ} \mathrm{N}$ (Fig. 7b) and then extends equatorward reaching the maximum at lag 10 days near $400 \mathrm{hPa}$ and $70^{\circ} \mathrm{N}$ (Fig. 7c). As for the results for the two month averaging, it is the waves with zonal wavenumber 1-3 that contribute to the cross-correlation patterns based on the low-passed daily data in Figs. 6 and 7.

The same analysis for the LMDz GCM (not shown) also reveals a positive and significant tropospheric signal in $F^{(z)}$ after a NAM event, centred around $60^{\circ} \mathrm{N}$. It is also larger in amplitude than the negative tropospheric signal seen before. Nevertheless, the difference in amplitude between the $F^{(z)}$ anomalies occurring after and before NAM events is not as pronounced as it is in the reanalysis dataset. As for the reanalysis the tropospheric signals in the LMDz GCM are associated with the planetary waves 1-3.

When a time-series drives in part the changes in a second one (like here the tropospheric $\left\langle F^{(z)}\right\rangle$ driving the NAM index) it is quite normal to find a correlation of opposite sign between the second series and the first at negative and positive lags. These does not necessarily witness that there is feedback of the second series on the first one (see for instance von Storch and Zwiers, 1999, p. 238). What is more meaningful in our context, is that in the troposphere, the positive anomalies at positive lags in Figs. 6 and 7 are much larger in amplitude than the negative anomalies found at negative lags. Accordingly, and providing that this difference in amplitude between the tropospheric values of $\left\langle F^{(z)}\right\rangle$ is significant, it is this difference in amplitude that we will interpret as a feedback of the stratosphere on the tropospheric wave forcing.

To test whether these differences are significant, we next consider the lagged regression of the vertical EP flux over $45^{\circ}-75^{\circ} \mathrm{N}\left\langle F^{(z)}\right\rangle$ onto the 20 -hPa NAM index. We choose to test the lagged regression in the stratosphere at $100 \mathrm{hPa}$, and in the troposphere at $400 \mathrm{hPa}$. We also include in the test, the lagged regression between the EP flux $F^{(z)}$ and the $20-\mathrm{hPa}$ NAM at one point $\left(400 \mathrm{hPa}, 70^{\circ} \mathrm{N}\right)$ where the strongest positive cross correlations are found in Fig. 7c. For the lagged regressions chosen (see Fig. 8), we identify the positive lag at which the regression is maximum and the negative lag at which it is minimum. We then take values of the regression at these negative $\left(r_{\min }\right)$ and positive $\left(r_{\max }\right)$ lags for each year. Two samples $R_{\min }$ and $\mathrm{R}_{\max }$ are not independent of each of other, since the positive values follow the negative ones during the NAM life cycle. Therefore we apply a one-side paired difference $t$-test with the null hypothesis $H_{0}: \overline{\left|\boldsymbol{R}_{\max }\right|-\left|\boldsymbol{R}_{\min }\right|} \leq 0$.

The estimated $\mathrm{p}$-values in Table 1 give a strong evidence to reject the null hypothesis confirming that for the NCEPNCAR reanalysis the tropospheric EP flux anomalies after a NAM event are significantly larger in amplitude than those before, especially in the polar region. However, there is no evidence that an increase in the tropospheric EP flux caused by the stratospheric circulation has a corresponding increase in the stratosphere. The same test applied to the LMDz GCM results shows that the difference between positive and negative lags is less significant in the troposphere. The most significant result is for the regression between the $20-\mathrm{hPa}$ NAM and the $500-\mathrm{hPa}\left\langle F^{(z)}\right\rangle$, where the difference between the maximum regression at positive lag and the minimum regression at negative lag, is significant at the $15 \%$ level (not shown). 


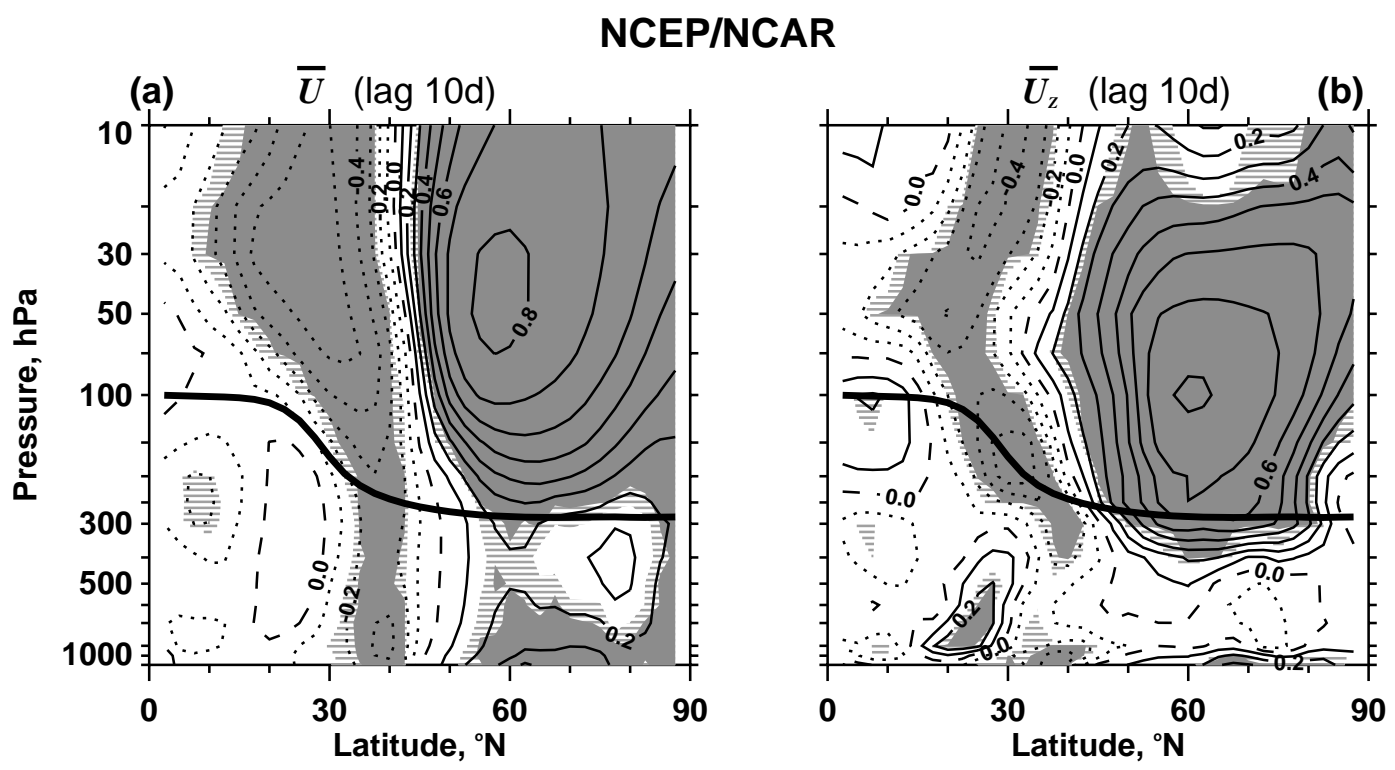

Fig. 9. Cross correlation of the 60-day low-passed NAM index at $20 \mathrm{hPa}$ with the 60-day low-passed anomalies of (a) zonal mean zonal wind and (b) zonal mean zonal wind shear at lag 10 days. Contours and shading as in Fig. 2. The NCEP-NCAR reanalysis.

Table 1. Minimum and maximum lagged regression between the 60-day low-passed vertical EP flux anomalies and the 60-day lowpassed normalized NAM index at $20 \mathrm{hPa}\left[10^{4} \mathrm{~kg} \mathrm{~m}^{-1} \mathrm{~s}^{-2}\right]$, lag in days, and p-values for the one-side paired difference $t$-test with the null hypothesis $H_{0}: \overline{\left|\boldsymbol{R}_{\max }\right|-\left|\boldsymbol{R}_{\min }\right|} \leq 0$. The NCEP-NCAR reanalysis.

\begin{tabular}{cccc}
\hline EP flux & $\mathrm{R}_{\min }(\mathrm{lag})$ & $\mathrm{R}_{\max }(\mathrm{lag})$ & $\mathrm{p}$-value \\
\hline $45^{\circ}-75^{\circ} \mathrm{N}, 100 \mathrm{hPa}$ & $-1.67(-19)$ & $1.44(27)$ & 0.988 \\
$45^{\circ}-75^{\circ} \mathrm{N}, 400 \mathrm{hPa}$ & $-1.91(-35)$ & $3.27(16)$ & 0.001 \\
$70^{\circ} \mathrm{N}, 400 \mathrm{hPa}$ & $-2.62(-43)$ & $4.69(11)$ & $<0.001$ \\
\hline
\end{tabular}

\section{Discussion}

In the past, at least two dynamical mechanisms have been proposed to explain the tropospheric planetary waves response to the state of the stratosphere. We will next analyse if the large-scale conditions these mechanisms request are satisfied in the frequency domain we have focused on.

The first mechanism is the modulation of the quasistationary planetary waves by the zonal mean state. It is related to the fact that in the troposphere, the amplitude of quasi-stationary planetary waves is a function of the amplitude of the zonal wind $\bar{u}$ and that even a small change in $\bar{u}$, can lead to large changes in the amplitude of the quasistationary waves (Branstator, 1984; Nigam and Lindzen, 1989; Kang, 1990; Ting et al., 1996; DeWeaver and Nigam, 2000). In this framework, stronger than usual $\bar{u}$ causes an increase in the amplitude of the quasi-stationary waves. Pro- viding that the downward propagation also influences the tropospheric $\bar{u}$ anomalies (see Kodera et al., 1990; Christiansen, 2001), we can expect this to be at work in our case as well. This point is illustrated in Fig. 9a which presents a cross correlation between the 60-day low-passed 20-hPa NAM index and the $\bar{u}$ anomalies at lag 10 days, e.g. when the maximum of the cross correlations between the NAM and the vertical EP flux was found in the polar troposphere (see Fig. 7c). Although there is no coherence in the middle and upper polar troposphere, a significant coherence is evident in the lower troposphere north of $50^{\circ} \mathrm{N}$. As the modulation of the planetary wave forcing is strongly controlled by the low-level winds (Held and Ting, 1990) the Fig. 9a is consistent with the picture that stratospheric changes can affect, at a later stage, the tropospheric quasi-stationary wave forcing.

The second mechanism is related to the fact that the planetary-scale baroclinic instability in the troposphere are modulated by changes in the lower stratospheric vertical wind shear $\bar{u}_{z}$. Wittman et al. (2007), for instance, have shown that an increased $\bar{u}_{z}$ in the lower stratosphere increases the growth rates of baroclinic modes with synoptic wavenumbers 4-7. At the same time, the planetaryscale modes of baroclinic instability can also be more unstable although they grow more slowly than the synoptic ones (Tanaka and Tokinaga, 2002). If we recall that during winter, the wave-driven low-frequency anomalies in the lower stratosphere have long persistence (Baldwin et al., 2003) and can potentially influence baroclinic instability growing on timescales near a month, this mechanism can also be at work to explain our results. To check that it is indeed the case, Fig. $9 \mathrm{~b}$ shows that at periods longer than 60 days, the stratospheric $\bar{u}_{z}$ anomalies are strongly correlated with the $20-\mathrm{hPa}$ 
NAM index, and that the signal even penetrates into the upper troposphere. This result is also consistent with recent model studies which demonstrate that high-latitude planetary wave baroclinic instabilities are more unstable when the polar vortex is stronger (Tanaka and Tokinaga, 2002; Song and Robinson, 2004). One more point in our study which is also in agreement to the model results in Tanaka and Tokinaga (2002) and Song and Robinson (2004) is that the troposheric planetary wave response we identify is essentially confined to the polar troposphere (Fig. $7 \mathrm{~b}$ and $\mathrm{c}$ here).

\section{Summary}

The results of this study suggest that in the low-frequency band of the downward propagation an anomalous state of the stratosphere modulates the tropospheric planetary wave signal. By means of the cross-spectral analysis, it is shown for the first time that that the downward propagation of stratospheric anomalies only occurs at periods longer than 50-60 days, which justifies the choice of various ad-hoc low-pass filters used in previous studies (e.g., Kodera et al., 1990; Baldwin and Dunkerton, 1999; Christiansen, 2001; Karpetchko and Nikulin, 2004). Moreover, our results suggest that the 60-day low-pass filter applied in the study presents an optimal choice to isolate the low-frequency downward propagating part of the stratospheric anomalies from the higherfrequency part which is in phase throughout the stratosphere, i.e. shows no downward propagation.

The stratospheric NAM index approximating the variability of the zonal mean state and the vertical EP flux anomalies from the stratosphere and the troposphere are significantly related with each other, at the periods of the downward propagation. At these periods, the EP flux and the NAM are almost in lead-lag quadrature: the low-frequency EP flux anomalies of opposite sign about NAM events drive the onset and the subsequent decay of NAM events. This coincidence of characteristic frequencies for the downward propagation and for the high coherence between the NAM and the EP flux in the stratosphere clearly shows that the downward propagation in the stratosphere is the result of wave-mean flow interaction at low frequencies.

There is also a remarkable effect in the troposphere and in the same low-frequency band as indicated above. The tropospheric EP flux anomalies coherent with the stratospheric NAM index are larger in amplitude after 7-10 days following a NAM event than at any time before. Taken into account that in a simple stochastic system without feedback forcing anomalies are equal around response extremes, at periods longer than 50-60 days, a stronger (weaker) polar vortex leads to a subsequent increase (decrease) in the upward EP flux in the troposphere. At the same time there is no evidence of a corresponding increase in the upward EP flux in the stratosphere. The response of the tropospheric upward EP flux to the stratospheric conditions is confined in the northern midlatitudes and polar regions and related to waves with zonal wavenumbers $1-3$ and, witnessing that there is a stratospheric influence on the low-frequency planetary waves in the troposphere.

In many aspects the stratospheric version of the $\mathrm{LMDz}$ GCM (Lott et al., 2005) shows results similar to the results based on the reanalysis. The model closely reproduces the characteristic frequencies of the downward propagation in the stratosphere (longer than 50-60 days) and the coherence and phase between the stratospheric NAM and the EP fluxes both in the stratosphere and troposphere. At the same time although the model also has a tendency to present tropospheric planetary wave response to the stratospheric anomalies as in the reanalysis the signature of the response is weaker and not so pronounced.

Acknowledgements. The authors thank the Climate Prediction Center for providing the NCEP-NCAR reanalysis data and ECMWF for providing the ERA-40 reanalysis. Alexey Karpechko is thanked for helpful suggestions and discussions. We also thank two reviewers for their helpful comments.

Topical Editor F. D'Andrea thanks D. Peters and another anonymous referee for their help in evaluating this paper.

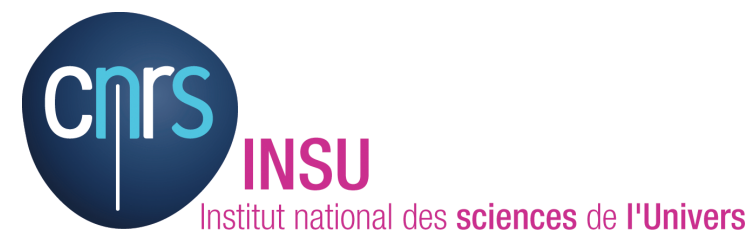

The publication of this article is financed by CNRS-INSU.

\section{References}

Ambaum, H. P. and Hoskins, B. J.: The NAO tropospherestratosphere connection, J. Climate, 15, 1969-1978, 2002.

Andrews, D. G., Holton, J. R., and Leovy, C. B.: Middle Atmosphere Dynamics, Academic Press, 489 pp., 1987.

Baldwin, M. P. and Dunkerton, T. J.: Propagation of the Arctic Oscillation from the stratosphere to the troposphere, J. Geophys. Res., 104, 30937-30946, 1999.

Baldwin, M. P., Stephenson, D. B., Thompson, D. W. J., Dunkerton, T. J., Charlton, A. J., and O'Neill, A.: Stratospheric memory and skill of extended-range weather forecasts, Science, 301, 636-640, 2003.

Baldwin, M. P. and Thompson, D. W. J.: A critical comparison of stratosphere-troposphere coupling indices, Q. J. Roy. Meteorol. Soc., 135, doi:10.1002/qj.407, 2009.

Black, R. X.: Stratospheric forcing of surface climate in the Arctic oscillation, J. Climate, 15, 268-277, 2002.

Branstator, G.: The relationship between zonal mean flow and quasi-stationary waves in the midtroposphere, J. Atmos. Sci., 41, 2163-2178, 1984.

Charney, J. G. and Drazin, P. G.: Propagation of planetary-scale disturbances from the lower into the upper atmosphere, J. Geophys. Res., 66, 83-109, 1961. 
Christiansen, B.: Stratospheric vacillations in a general circulation model, J. Atmos. Sci., 56, 1858-1872, 1999.

Christiansen, B.: Downward propagation of zonal mean zonal wind anomalies from the stratosphere to the troposphere: Model and reanalysis, J. Geophys. Res., 106, 27307-27322, 2001.

Coughlin, K. and Tung, K. K.: Tropospheric wave response to decelerated stratosphere seen as downward propagation in northern annual mode, J. Geophys. Res., 110, D01103, doi:10.1029/2004JD004661, 2005.

DeWeaver, E. and Nigam, S.: Zonal-eddy dynamics of the North Atlantic oscillation, J. Climate, 13, 3893-3914, 2000.

Dickinson, R. E.: Planetary Rossby waves propagating vertically through weak westerly wind wave guides, J. Atmos. Sci., 25, 984-1002, 1968.

Edmon, H. J., Hoskins, B. J., and McIntyre, M. E.: Eliassen- Palm cross section for the troposphere, J. Atmos. Sci., 37, 2600-2616, 1980.

Haynes, P. H., McIntyre, M. E., Shepherd, T. G., Mark, C. J., and Shine, K. P.: On the "downward control" of extratropical circulation by eddy-induced mean zonal forces, J. Atmos. Sci., 48, 651-680, 1991.

Held, I. M. and Ting, M.: Orographic versus thermal forcing of stationary waves: the importance of the mean low-level wind, J. Atmos. Sci., 47, 495-500, 1990.

Holton, J. R. and Mass, C.: Stratospheric vacillation cycles, J. Atmos. Sci., 33, 2218-2225, 1976.

Kalnay, E., Kanamitsu, M., Kistler, R., Collins, W., Deaven, D., et al.: The NCEP/NCAR 40-year reanalysis project, B. Am. Meteorol. Soc., 77, 437-471, 1996.

Kang, I.-S.: Influence of zonal mean flow change on stationary wave fluctuations, J. Atmos. Sci., 47, 141-147, 1990.

Karpetchko, A. and Nikulin, G.: Influence of early winter upward wave activity flux on midwinter circulation in the stratosphere and troposphere, J. Climate, 17, 4443-4452, 2004.

Kodera, K., Yamazaki, K., Chiba, M., and Shibata, K.: Downward propagation of upper stratospheric mean zonal wind perturbation to the troposphere, Geophys. Res. Lett., 17, 1263-1266, 1990.

Limpasuvan, V., Thompson, D. W. J., and Hartman, D. L.: The lifecycle of the Northern Hemisphere sudden stratospheric warming, J. Climate, 17, 2584-2596, 2004.

Limpasuvan, V., Hartman, D. L., Thompson, D. W. J., Jeev, K., and Yung, Y. L.: Stratosphere-troposphere evolution during polar vortex intensification, J. Geophys. Res., 110, D24101, doi:1029/2005JD006302, 2005.

Lott, F., Fairhead, L., Hourdin, F., and Levan, P.: The stratospheric version of LMDz: dynamical climatologies, arctic oscillation, and impact on the surface climate, Clim. Dynam., 25, 851-868, doi:10.1007/s00382-005-0064-x, 2005.

Newman, P. A., Nash, E. R., and Rosenfield, J. E.: What controls the temperature of the Arctic stratosphere during the spring?, J. Geophys. Res., 106, 19999-20010, 2001.
Nigam, S. and Lindzen, R. S.: The sensitivity of stationary waves to variations in the basic state zonal flow, J. Atmos. Sci., 46, 1746$1768,1989$.

Nikulin, G. and Karpechko, A.: The mean meridional circulation and midlatitude ozone buildup, Atmos. Chem. Phys., 5, 31593172, 2005, http://www.atmos-chem-phys.net/5/3159/2005/.

Perlwitz, J. and Harnik, N.: Observational evidence of a stratospheric influence on the troposphere by planetary wave reflection, J. Climate, 16, 3011-3026, 2003

Plumb, R. A. and Semeniuk, K.: Downward migration of extratropical zonal wind anomalies, J. Geophys. Res., 108, 4223, doi:10.1029/2002JD002773, 2003.

Polvani, L. M. and Waugh, D. W.: Upward wave activity flux as precursor to extreme stratospheric events and subsequent anomalous surface weather regimes, J. Climate, 17, 3548-3554, 2004.

Schoeberl, M. R.: A study of stratospheric vacillations and sudden warmings on a $\beta$-plane. Part I: Single wave-mean flow interaction, J. Atmos. Sci., 40, 769-787, 1983.

Scott, R. K. and Polvani, L. M.: Stratospheric control of upward wave flux near the tropopause. Geophys. Res. Lett., 31, L02115, doi:10.1029/2003GL017965, 2004.

Smith, S.: Digital Signal Processing: A Practical Guide for Engineers and Scientists. Newnes, 672 pp., 2002.

Song, Y. and Robinson, W. A.: Dynamical mechanism for stratospheric influence on the troposphere, J. Atmos. Sci., 61, 17111725, 2004.

Tanaka, H. L. and Tokinaga, H.: Baroclinic instability in high latitudes induced by polar vortex: a connection to the Arctic oscillation, J. Atmos. Sci., 59, 69-82, 2002.

Thede, L: Practical Analog And Digital Filter Design, Artech House, 270 pp., 2004.

Thompson, D. W. J., Baldwin, M. P., and Wallace, J. M.: Stratospheric connection to Northern hemisphere wintertime weather: implications for prediction, J. Climate, 15, 1421-1428, 2002.

Thompson, D. W. J., Furtado, J. C., and Shepherd, T. J.: On the tropospheric response to anomalous stratospheric wave drag and radiative heating, J. Atmos. Sci., 63, 2616-2629, 2006.

Ting, M., Hoerling M. P., Xu, T., and Kumar, A.: Northern Hemisphere teleconnection patterns during extreme phases of zonalmean circulation, J. Climate, 9, 2614-2633, 1996.

Uppala, S. M., Kållberg, P. W., Simmons, A. J., Andrae, U., Da Costa Bechtold, V., et al.: The ERA-40 re-analysis, Q. J. Roy. Meteorol. Soc., 131, 2961-3012, 2005.

von Storch, H. and Zwiers, F. W.: Statistical Analysis in Climate Research, Cambridge University Press, 484 pp., 1999.

Wittman, M. A. H., Polvani, L. M., Scott, R. K., and Charlton, A. J.: Stratospheric influence on baroclinic lifecycles and its connection to the Arctic oscillation, Geophys. Res. Lett., 31, L16113, doi:10.1029/2004GL020503, 2004.

Wittman, M. A. H., Charlton, A. J., and Polvani, L. M.: The effect of lower stratospheric shear on baroclinic instability, J. Atmos. Sci., 64, 479-496, 2007. 